\title{
The Design and Analysis of the Bionic Ostrich Biped Robots
}

\author{
SONG Zhong-Kang ${ }^{1}, \mathrm{JING}_{\mathrm{Q}}{ }^{1}, \mathrm{CHEN}_{\mathrm{Ya}} \mathrm{Ning}^{1}{ }^{\text {and TANG Dong-Hong }}{ }^{1}$ \\ ${ }^{1}$ Department of mechanical engineering, The Academy of Armored Forces Engineering, Beijing 100072 , China
}

\begin{abstract}
In this paper,the ostrich as the research object, using the related mechanism ,according to the motion characteristics of biped walking upright, bionic design of its leg, foot structure, and computer modeling, finite element analysis and motion simulation , to achieve walking, running, jumping, to form of motion.
\end{abstract}

The biped robots have the advantages of low requirement to pedestrian environment,fitness high ground,flexible walking and covering a small area of walking system,large range of activities,there is the broad application prospect in military field.The most biped robots are humanoid robots at present,ostrich is a kind of rare animal what use biped to walk in nature and has the feature of fast running,strong obstacle ability and agile motion.It is a high application value of studying and simulating design. The article proposes a design project of biped robots to simulate the ostrich's legs and feet structure,uses INVENTOR to come true the motion simulation and 3D modeling.

\section{The project design}

\subsection{The structure of ostrich leg bone}

The ostrich's waist and a part of hind legs are very strong for adapting the run and supporting the weight.The ostrich's hind leg bone is very strong. The waist(as figure 1 ) is made up of several bones including ischium,pubis and ilium ,these bones form pelvis. The ilium,located in the left and right side of the synsacrum,is a slice of flat bone.The ischium what lies behind and below the ilium is also a slice of flat bone.There is the holes of ischium.The pubis,located in the ventral edge of the ischium,is a piece of long and thin bone.The hind leg includes the thighbone(associated with acetabulum),the tibiotarsus(a thick long bone,the knee lies in the joint of the tibiotarsus and the thighbone),the fibula(lies in the ouside of the tibiotarsus,is a thin,small and long bone), the tarsometatarsus(a piece of long bone healed by tarsal and phalanx)and the phalanx ${ }^{[1]}$.

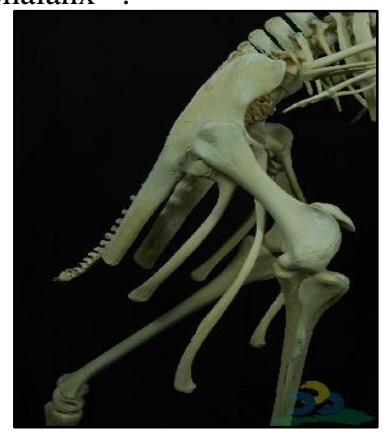

Figure 1. The ostrich's waist and a part of hind legs

\subsection{The leg design}

We mainly design the leg instructure of the bionic ostrich biped robots according to the study of ostrichs'bone.The ostrich leg instructure is divided into the thighbone,the tibiotarsus and the tarsometatarsus(part).This bionic robots simulate the way of ostrich's action.There are three joints and three degrees of freedom on each leg,so the whole has six degrees of freedom,as shown in the figure 2.This design omits pubis for simplifying the form of instructure and instead of the high-strength and stiffness "blade point".

It is drived by the way of mixing the servo motor and the hydraumatic. The main driving force supported by the high torque and the high precision produces the principal rotating motion by the periodic rotation angle how the two sides of servo motors drive the thighbone.Two sets of hydraulic equipment support the supplementary motor.Between the thighbone and the tibiotarsus and the tarsometatarsus(part) has a hydraulic device,as shown in the figure 3.The angel between the thighbone and the tibiotarsusand and the tarsometatarsus(part) is changed by adjusting hydraulic shaft,combines the principal rotating motion to complete the action.

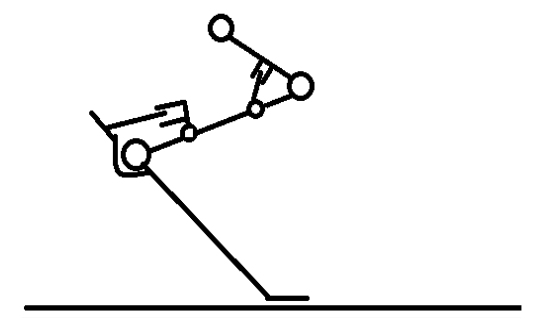

Figure 2. Design of the leg

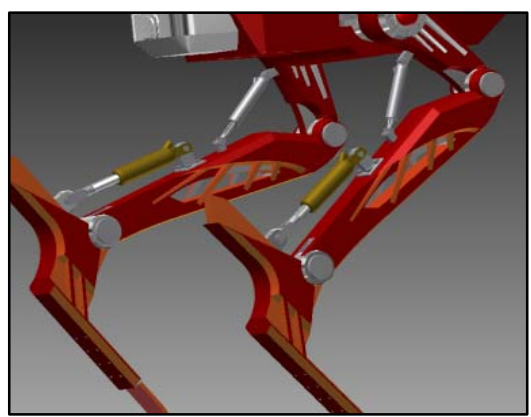

Figure 3. The bionic structure of ostrich leg 


\subsection{Solar wing structure design}

Solar wing is setted in the back of the ostrich bionic robot, income usually folding solar wing its sheath, to add electricity solar wing unfolded into a half-circle, doing their utmost receive sun to obtain energy recharge the built-in control circuit power supply;Another function of solar wing is used to maintain the balance of the ostrich bionic robot, when the robot running in high speed, balance control just close to the coordination of the legs is not enough, so we can design deflection, and can be adjusted up and down small solar sails, by wing contraction and adjust the up and down and then adjust the bionic ostrich's center of gravity and balance.

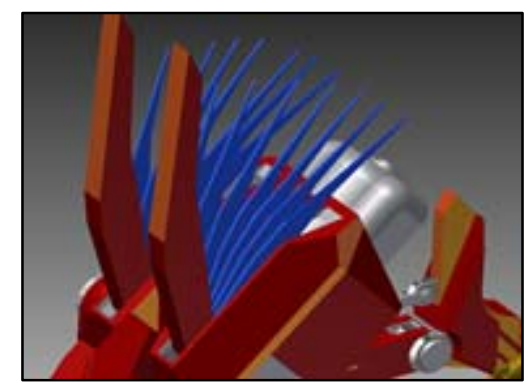

Figure 4. The solar wing

\subsection{The head comprehensive detection device structure design}

Ostrich bionic robot can freely adjustable head, and through the mechanical structure design makes it with two degrees of freedom, free space to any Angle deflection and thus achieve its claim to receive 360 degree information.Ostrich head bionic robot equipped with built-in high camera to observe and record the task execution and surrounding environment.Built-in infrared and thermal detector makes the ostrich bionic robot's ability to get nocturnal, greatly improve the timeliness and spatiality of its activities.The back with a built-in audio positioning collector, this device can through the surrounding environment inside the audio signal from the target to perceive and positioning, to found the shot point and the location of the enemy sniper, and real-time transmit the information to the combat team, actively cooperate with other power carries out operations ${ }^{[2]}$.

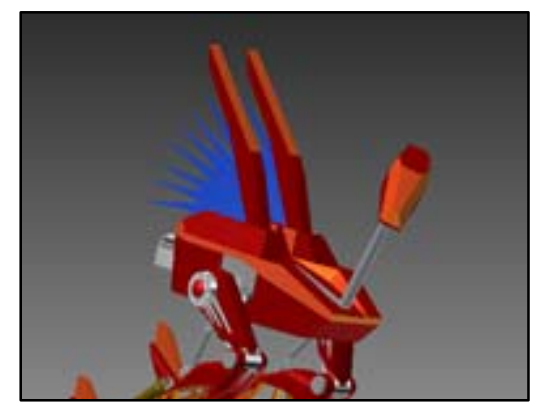

Figure 5. The head comprehensive detection device

\section{The finite element analysis}

The finite element analysis is mainly aimed at stress bearing larger agencies and parts in the mechanical structure, through the calculation analysis of the stress and material properties can get parts at the time the working state of the environment, such as various degrees of freedom on the stress, deformation and the stress of the whole parts, safety coefficient and so on.

\subsection{The motor shaft}

The motor shaft uses a material of the high strength alloy steel, under the environment of outside force of $5000 \mathrm{~N}$ on the stress analysis, the following results are obtained:

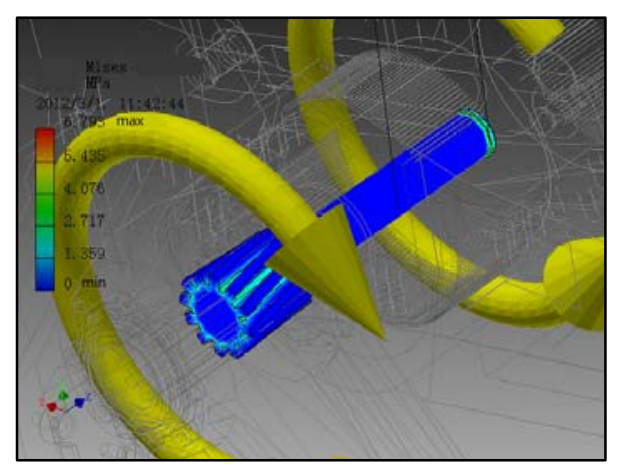

Figure 6. Mises equivalent stress

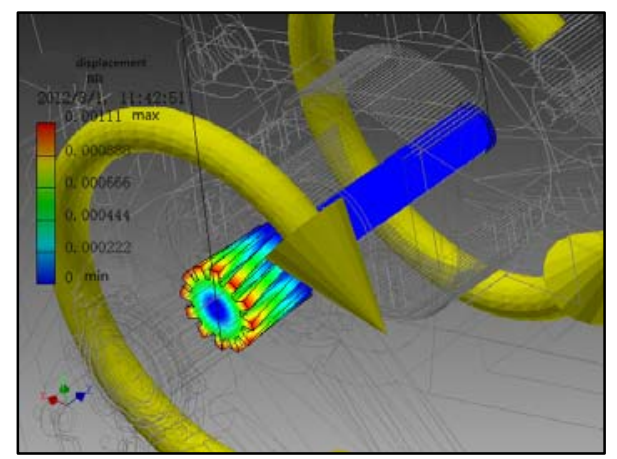

Figure 7. Displacement

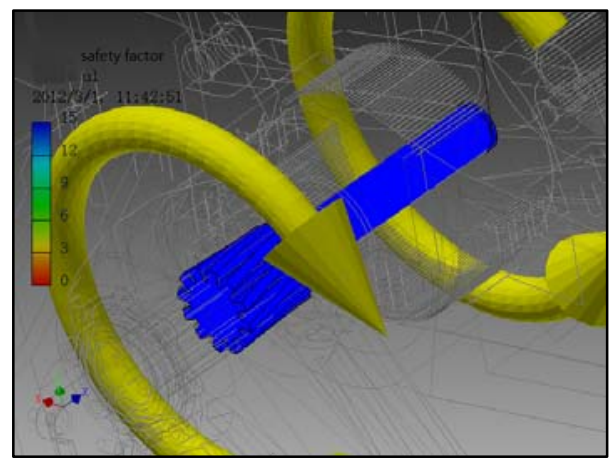

Figure 8. Safety factor 


\subsection{The blade point}

Blade is the key part of bionic ostrich movement, demanding it must have a high yield strength, so choose high-performance carbon fiber fiber composite material, will produce a larger displacement under great external force but can ensure the safe and stable, the image below for the material under external force of $700 \mathrm{n}$ performance show, displacement is great, but the factor of safety is good.

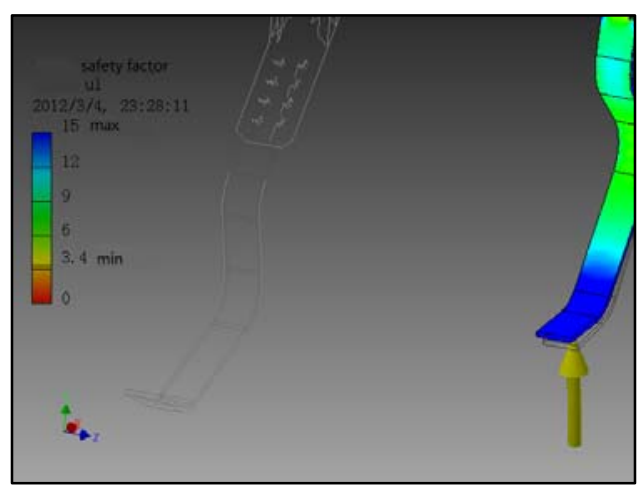

Figure 9. Mises equivalent stress

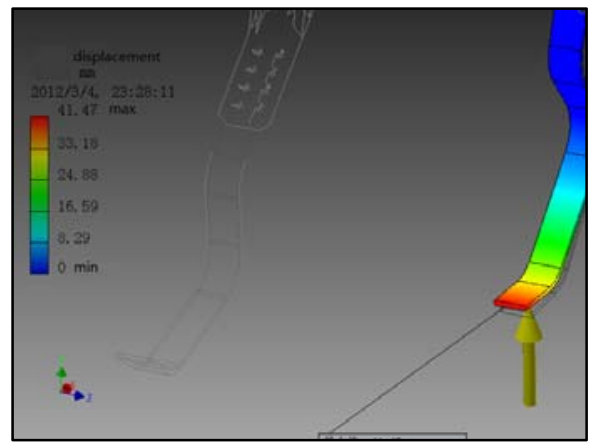

Figure 10. Displacement

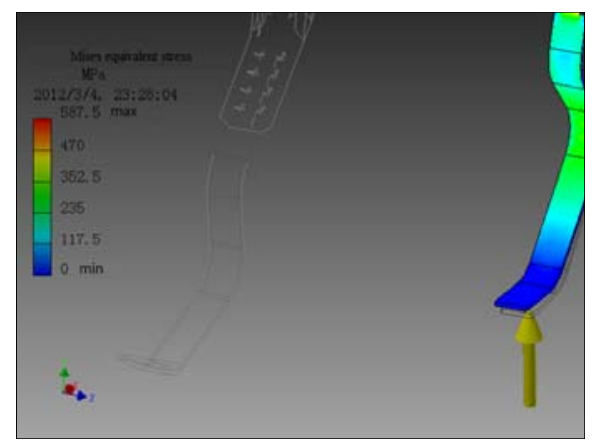

Figure 11. Safety factor

\section{Motion simulation and analysis}

We use INVENTOR to build the 3D model,enter the INVENTOR/SIMULATION simulation module,add motion constraints and drive.The thighbone and the tibiotarsus use servo motor and hydraulic straight motion,the curve of simulation as shown in figure 12 and 13.The simulation results shows that the robot motion leg swing range within the scope of the drive control, coherent motion, good coordination, for the design of the control system laid a good foundation ${ }^{[3]}$.

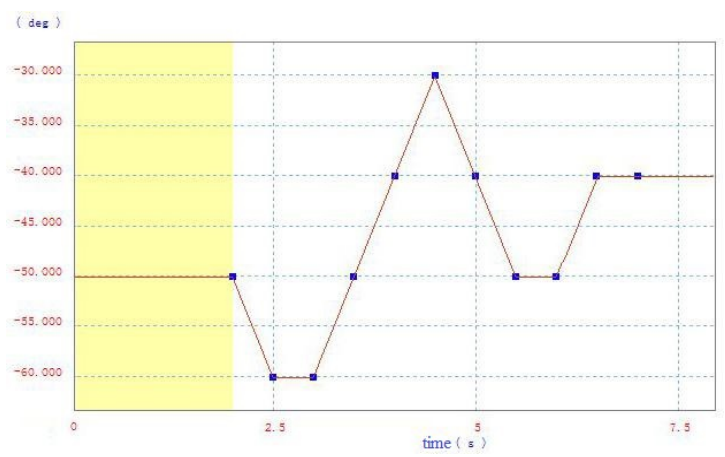

Figure 12. Femoral movement simulation curve

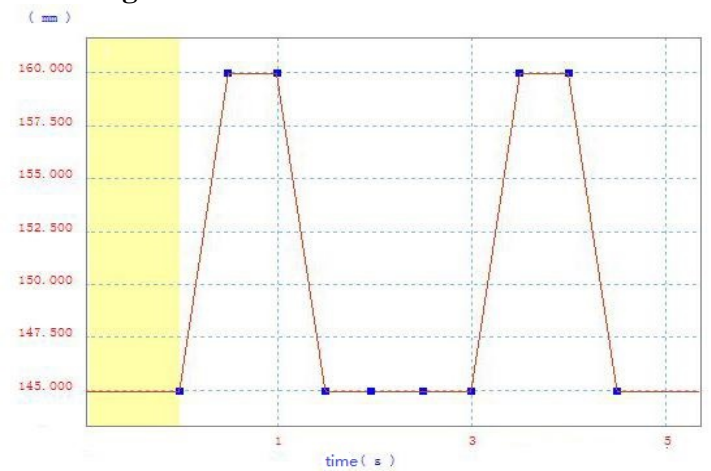

Figure 13. Tibiotarsus movement simulation curve

\section{Conclusion}

Bionic ostrich bipedal robot driven by motor and servo hydraulic hybrid mode, in the guarantee accurate provide a fixed Angle of movement at the same time provides a strong torque and support.The bionic design of ostrich's innovation point is not limited to the real ostrich legs every bionic structure, but the use of simple mechanical structure -"blade", instead of ostrich leg tarsometatarsus (part), and all of the phalanges, combined with the femoral and tibial tarsi coordinated movement of the finish.The simulation results show that this design has simple structure, reliable movement, good coordination, easy to control.

\section{References}

[1] Yao Jiyuan,Sun Zewei,Jiang Huaizhi.The ostrich bone observation[J].2006,1.

[2] Xu Kai.Humanoid robot gait planning algorithm and implementation[D],Beijing:TsinghuaUniversity,2004.

[3] Yang Yiming.Biped walking robots simulation [D].Beijing:Tsinghua University,2005,6. 